\section{SCHLECHTE PROGNOSE}

\section{Bei vier von zehn Frauen bleibt der Herzinfarkt stumm}

\author{
- Ein Myokardinfarkt macht sich bei \\ Frauen öfter als bei Männern nicht durch \\ Thoraxschmerzen bemerkbar. Dies zeigt \\ eine Studie, die Daten von 1143513 In- \\ farktpatienten, darunter 481581 Frauen, \\ ausgewertet hat. Die Patienten waren zwi- \\ schen 1994 und 2006 im National Registry \\ of Myocardial Infarction erfasst worden. \\ Frauen, die wegen eines Herzinfarkts ein \\ Krankenhaus aufsuchten, waren im Mittel \\ gut sieben Jahr älter als ihre männlichen \\ Leidensgenossen (73,9 vs. 66,5 Jahre). $42 \%$ \\ von ihnen berichteten nicht über Schmer- \\ zen oder Engegefühl im Brustbereich. Von \\ den Männern stellten sich nur 30,7\% ohne \\ Thoraxschmerzen vor. Diese geschlechts- \\ spezifische Differenz war bei den unter \\ 45-Jährigen am stärksten und ging mit
}

dem Alter zurück. In der Altersgruppe über 75 fehlte das Leitsymptom bei ungefähr der Hälfte der Frauen wie der Männer.

Frauen starben häufiger als Männer noch in der Klinik (14,6\% vs. 10,3\%). Generell war die Mortalität erhöht, wenn anfangs keine Thoraxschmerzen bestanden hatten. Für jüngere Frauen war das Fehlen des Leitsymptoms jedoch verhängnisvoller als für Männer desselben Alters - mit einer Sterberate von $14,5 \%$ gegenüber $10,6 \%$ bei den 45- bis 54-Jährigen. Auch diese Geschlechterdifferenz nahm mit dem Alter ab. Für die schlechtere Überlebenschancen von Patienten ohne Thoraxbeschwerden machen die Autoren vor allem Begleiterkrankungen (Diabetes) und klinische Charakteristika des Herzinfarkts sowie die sel-

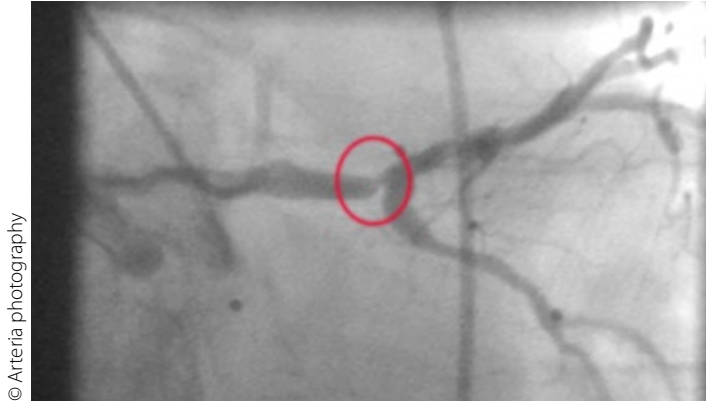

Hauptstammstenose. Gleiches Bild, unterschiedliche Symptome?

tenere Anwendung von Akutmaßnahmen verantwortlich. Die besonders schlechte Prognose von (jüngeren) Frauen sei wahrscheinlich auch auf Unterschiede in der Pathophysiologie zurückzuführen. Generell treten Herzinfarkte bei Frauen später auf, vermutlich wegen des Schutzes durch Östrogene. Wenn sie früher bertoffen sind, liegt möglicherweise ein besonders aggressives Krankheitsbild vor. BS =

\title{
ERNÄHRUNGSSTUDIE
}

\section{B-Vitamine und Omega-3-Fette schützen nicht vor Krebs}

- Französische Forscher haben untersucht, ob die Nahrungsergänzung mit BVitaminen und Omega-3Fettsäuren Karzinomen vorzubeugen hilft - mit negativem Ergebnis. Frauen könnte die Einnahme sogar gefährlich werden.

Für ihre Studie hatten Ernährungsepidemiologen um Valentina Andreeva, Paris, auf die Daten von 514 Frauen und 1987 Männern zurückgegriffen, die von

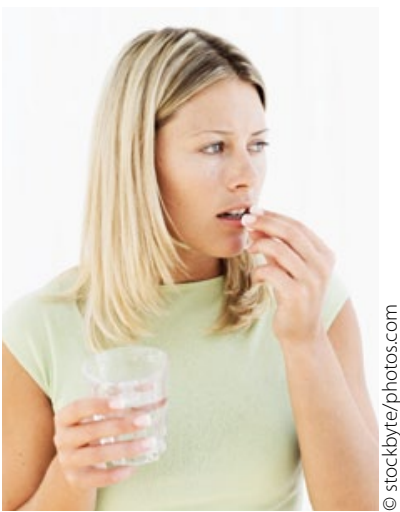

Nahrungsergänzung: mehr Schaden als Nutzen? vention kardiovaskulärer Ereignisse zu prüfen. Die Probanden schluckten fünf Jahre lang entweder - 0,56 mg 5-Methyl-Tetrahydrofolat plus $3 \mathrm{mg}$ Vitamin B6 plus 0,02 mg Vitamin B12 oder - 600 mg der Omega-3Fettsäuren Eicosapentaen- und Docosahexaensäure oder

- die B-Vitamine plus die Omega-3-Fettsäuren oder

- Placebo.

Die Autoren prüften nun, wie sich die Nahrungsergänzung auf die Krebsinzidenz auswirkte: Insgesamt erkrankten 7,3\% der Männer und 5,6\% der Frauen an Krebs. Dabei kristallisierte sich bei den Männern im Vergleich zu Placebo kein Zusammenhang mit den Supplementen heraus. Bei den Frauen erhöhte die Einnahme von Omega-
3-Fettsäuren hingegen das Krebsrisiko während der fünf Studienjahre um den Faktor 3. Auch bei den B-Vitaminen bestand eine Tendenz zu höherer Krebsinzidenz, allerdings war die Steigerung um den Faktor 2,18 nicht signifikant $(p=0,06)$.

Als Ursache für die erhöhte Krebsgefahr für Frauen vermuten die Forscher Einflüsse auf den Östrogenstoffwechsel.

RB -

Andreeva VA et al. Arch Intern Med 2012; DOI: 10.1001/ archinternmed.2011.1450

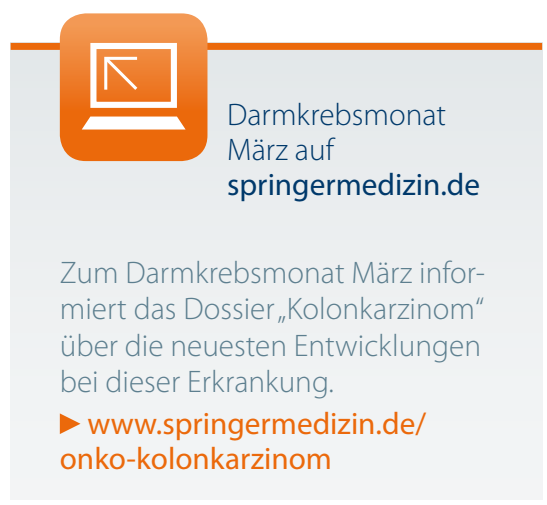

